\title{
A CLASSIFICAÇÃO ABC NA GESTÃO DE MATERIAIS DE CONSUMO EM UMA INSTITUIÇÃO FEDERAL DE ENSINO SUPERIOR
}

\section{ARTIGO ORIGINAL}

COSTA, Samuel Chagas da ${ }^{1}$

REIS, Augusto da Cunha ${ }^{2}$

COSTA, Samuel Chagas da. REIS, Augusto da Cunha. A classificação ABC na gestão de materiais de consumo em uma Instituição Federal de Ensino Superior. Revista Científica Multidisciplinar Núcleo do Conhecimento. Ano 05, Ed. 10, Vol. 15, pp. 34-47. Outubro de 2020. ISSN: 2448-0959, Link de acesso: https://www.nucleodoconhecimento.com.br/engenharia-deproducao/classificacao-abc

\section{RESUMO}

O sucesso qualquer organização depende de uma gestão eficiente de todos os seus recursos, inclusive os recursos de materiais. Assim, gestão de materiais de consumo compõe as estratégias das organizações, uma vez que materiais estocados significam capital ocioso investido no almoxarifado. Este trabalho tem como objetivo classificar os materiais de consumo de maior importância para a gestão do almoxarifado em uma instituição federal de ensino superior, e para isso o trabalho utilizou a classificação $A B C$ de materiais, ou curva de Pareto, a qual classifica os itens estocados quanto ao seu valor de aquisição. A metodologia de pesquisa é quantitativa, o universo de estudo é a própria instituição, e um dos campi serviu de amostra por acessibilidade, o qual também foi utilizado como pesquisa documental. Como resultado, o trabalho identificou 18 diferentes tipos de materiais de consumo, classificados como $\mathrm{A}$, dentre os 115 analisados. Concluiu-se que esses são os materiais em que a gestão deverá

\footnotetext{
${ }^{1}$ Pós-graduado em Gestão Pública, Graduado em Administração.

2 Orientador. Doutor em Engenharia de Produção.
} 
concentrar a máxima atenção, visto que eles consomem os maiores recursos financeiros destinados a esse elemento da despesa pública, ainda que em pequenas quantidades compradas.

Palavras-chave: Material de consumo, classificação ABC, compras públicas.

\section{INTRODUÇÂO}

Qualquer organização, pública ou privada, depende de uma gestão eficiente de todos os seus recursos, inclusive os recursos materiais, o que torna essa parte da administração um dos pontos estratégicos para as organizações. Gonçalves (2016) afirma que o planejamento e o controle da gestão de materiais são capazes de reduzir o investimento ocioso em estoque, melhorar o processo de compras e proporcionar vantagem competitiva ao produto ou ao serviço da empresa.

Feng et al. (2018) afirmam que o excesso de compras leva ao excesso de estoques e impede o uso eficiente de recursos financeiros. Para Halasz (2017), o cenário planejado não é atendido quando ocorrem erros na previsão da demanda, e consequentemente em todo o projeto de compras. Santos et al. (2018) asseveram que o planejamento deficiente da demanda gera compras em quantidades conflitantes com a realidade, o que afeta o atendimento das necessidades dos clientes. Para Losilla e Valente (2017), a curva ABC permite acompanhar o crescimento da demanda, o que proporciona a melhoria dos processos internos da empresa.

Segundo Eduardo Maehler (2004), a administração de materiais engloba um ciclo de operações interdependentes e correlatas, as quais incluem a previsão, a aquisição, o transporte, o recebimento, o armazenamento, a distribuição, a conservação e o controle de inventários. Segundo Vianna (2006), o objetivo da gestão de materiais é suprir as organizações com os materiais certos, no custo correto, no tempo apropriado e nas qualidades adequadas. Para Dias (2016), a classificação ABC de materiais é importante para um controle eficaz dos estoques, uma vez que ela possibilita a adequada armazenagem dos suprimentos, bem como a maneira correta de operacionalizar o almoxarifado.

RC: 62236

Disponível em: https://www.nucleodoconhecimento.com.br/engenharia-de-producao/classificacao$\underline{a b c}$ 
O objetivo desta pesquisa é identificar os materiais de consumo mais importantes para a gestão de estoque em uma instituição pública de ensino superior, por meio da classificação $A B C$ de materiais, também conhecida como princípio de Pareto.

\subsection{AS COMPRAS PÚBLICAS}

$\mathrm{Na}$ administração pública, as compras públicas - também chamadas de elementos da despesa pública - são divididas em materiais permanentes e materiais de consumo. Aqueles englobam itens cuja duração ultrapassam os dois anos de vida útil, enquanto estes se limitam a produtos ou insumos com utilização de no máximo um biênio.

Os materiais de consumo se subdividem em diversos subitens, tais como: Material de expediente; Material de consumo de tecnologia da informação e comunicação; Material de manutenção bens imóveis e instalações; Ferramentas; Material elétrico e eletrônico; Material de proteção e segurança; dentre outros.

\subsection{ESTRUTURA DA PESQUISA}

Esta pesquisa está estruturada em 5 capítulos sendo este o introdutório. O segundo capítulo apresenta os principais aspectos teórico de forma a embasar conceitualmente a pesquisa. $O$ terceiro aborda o método utilizado para coleta de dados. $O$ quarto explora o resultado e as discussões sobre o estudo. O quinto externa as conclusões e considerações finais da pesquisa.

\section{FUNDAMENTAÇÃO TEÓRICA}

O presente capítulo pesquisa na doutrina os fundamentos para a boa compreensão do importante papel desempenhado pela gestão de materiais nas organizações, bem como para o entendimento da classificação $A B C$ de materiais. Para isso, o estudo utiliza artigos científicos de periódicos Capes entre os anos de 2015 e 2020 e bases como o Web of Science, Scopus e Scielo. 
De acordo com Santos et al. (2018), a existência de instrumentos e de procedimentos capazes de discorrer e amparar as tomadas de decisões é imprescindível para o gestor de materiais, uma vez que vários processos organizacionais serão impactados pelo resultado da tomada de decisão sobre o planejamento da demanda de materiais e insumos.

\subsection{A CLASSIFICAÇÃO DE MATERIAIS ABC}

A classificação $A B C$ ou princípio de Pareto é uma ferramenta utilizada para determinar quais itens devem ter prioridades na gestão do estoque de uma organização. De acordo com Jemelka, Chramcov e Kriz (2017), a técnica permite a separação das unidades em três classes, $A B C$, com diferentes níveis de relevância. A metodologia atribui máxima relevância para os itens de classe $A$, média relevância para os itens de classe $B$, e pequena relevância para os itens de classe $C$.

Ishizaka et al., (2018) afirmam que os materiais classificados como A possuem máxima relevância em relação a valores e exigem a máxima atenção do gestor de materiais. Entretanto, eles representam cerca de 10\% apenas do inventário total. Segundo os autores, os itens classificados como B, de média relevância em relação a valores, constituem $20 \%$ do armazenamento da organização. $E$, por último, os autores afirmam que os itens pertencentes à classificação $C$ são de pequena relevância em relação aos custos e à atenção do gestor e compõem $70 \%$ de todo o estoque da organização.

Zhao e Dong (2016) afirmam que distinguir a da importância ou prioridade dos materiais é o principal objetivo da curva $A B C$. Para os autores, os itens de classificação $A$, de pequena quantidade e maior valor empreendido, devem ser considerados como os mais importantes no controle do estoque. Para Amran e Fátima (2017), a gestão de materiais deve direcionar toda sua atenção aos itens de classificação $A$, visto que esses materiais possuem um alto valor empreendido e pequena quantidade estocada. 
Prajaksuwithee e Chutima (2019) afirmam que por meio da classificação ABC podese prever de demanda de materiais, implementar de uma política de reabastecimento, bem como evitar o excesso de estoques. Para Liu (2017), o modelo de classificação ABC permite o implemento de um plano de reposição automática de materiais, bem como ações de melhorias no processo de reabastecimento de suprimentos já existente na organização. Para Cedillo Campos (2015), a curva de Pareto permite não só a gestão de prioridades dos materiais estocados, bem como a análise na gestão de riscos do almoxarifado.

A classificação $A B C$, conforme mostra a figura 1 abaixo, identifica os materiais quanto ao seu valor e às suas quantidades.

Figura 1 - Classificação ABC

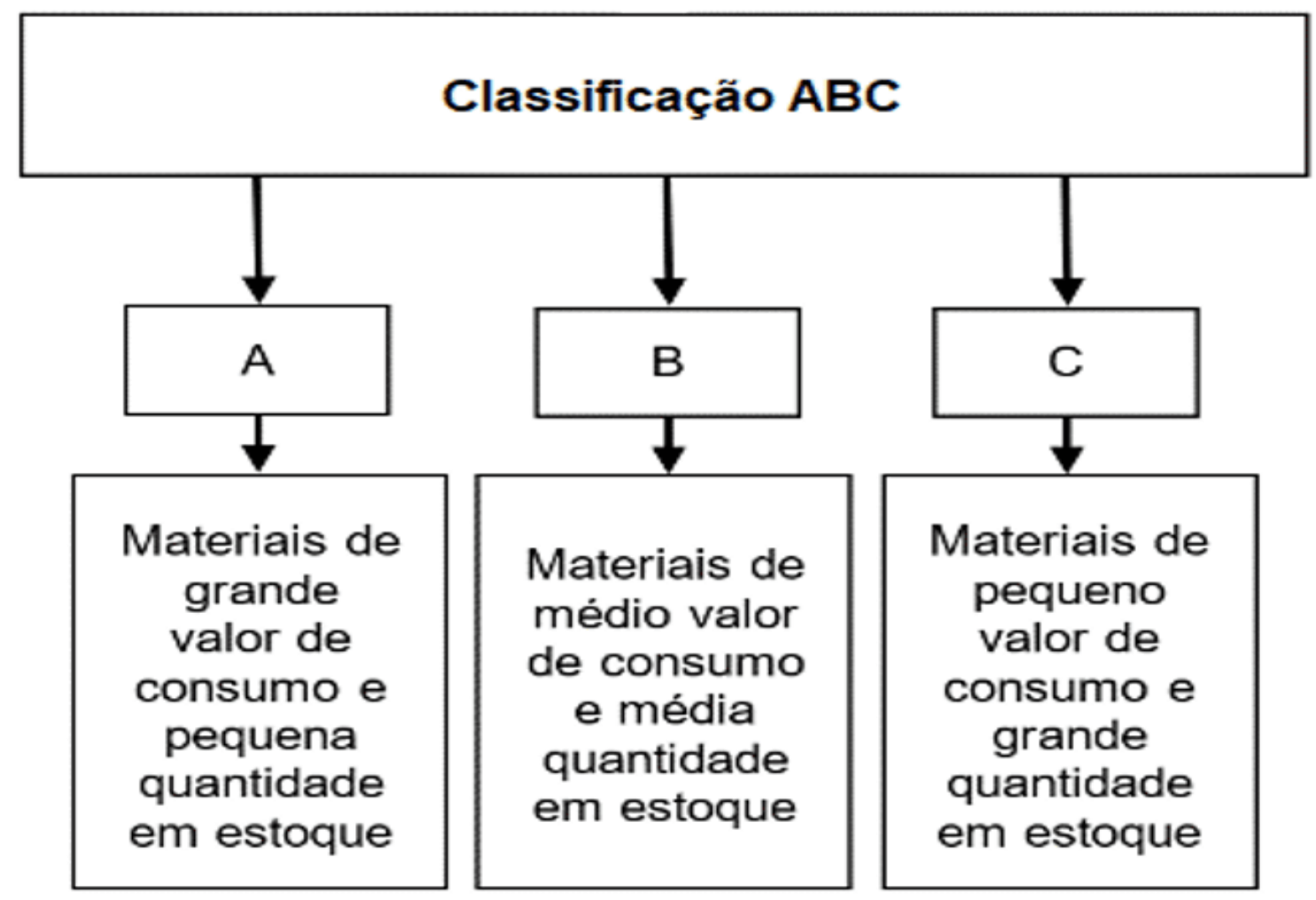

Fonte: elaborada pelos autores.

Para Amran e Fátima (2017), o setor de compras deve manter-se focado nos itens de classe $A$, os quais são materiais de elevado custo e alta utilização no processo de RC: 62236

Disponível em: https://www.nucleodoconhecimento.com.br/engenharia-de-producao/classificacao$\underline{a b c}$ 
ressuprimento. De acordo com os autores, são vários os procedimentos para a classificação $A B C$ de materiais, tais como: volume de uso por determinado tempo, o custo total de cada material por um dado período, o custo agregado e a relação de custo de uso pelo custo total.

Gonçalves (2016) afirma que os percentuais na classificação $A B C$ não são rigorosos. Segundo o autor, eles podem ser alterados conforme o perfil da curva $A B C$, bem como após uma consulta com especialistas de campo. Nesse sentido, Zenkova e Kabanova (2018) utilizaram as seguintes porcentagens para a classificação $A B C$ de materiais:

- Se a porcentagem acumulada $\leq 80 \%$, então o produto pertence ao grupo $A$;

- Se a porcentagem acumulada ficar entre $80 \%$ e $95 \%$, então o produto pertence ao grupo B;

- Se a porcentagem acumulada $>90 \%$, então o produto pertence ao grupo C.

Dessa forma, seguindo os parâmetros de Zenkova e Kabanova (2018), para uma classificação $A B C$, os itens de classe $A$ correspondem a $80 \%$ dos valores; os itens de classe $B$ a $15 \%$ dos valores; os itens de classe $C$ a $5 \%$ dos valores. Assim, o somatório da porcentagem individual deve ser igual a $100 \%$, bem como a porcentagem acumulada de um produto deve ser igual à soma de sua porcentagem individual com a porcentagem acumulada do produto anterior. A tabela 1 e o gráfico 1 mostram exemplos práticos utilizados por Zenkova e Kabanova (2018).

Tabela 1 - Classificação ABC

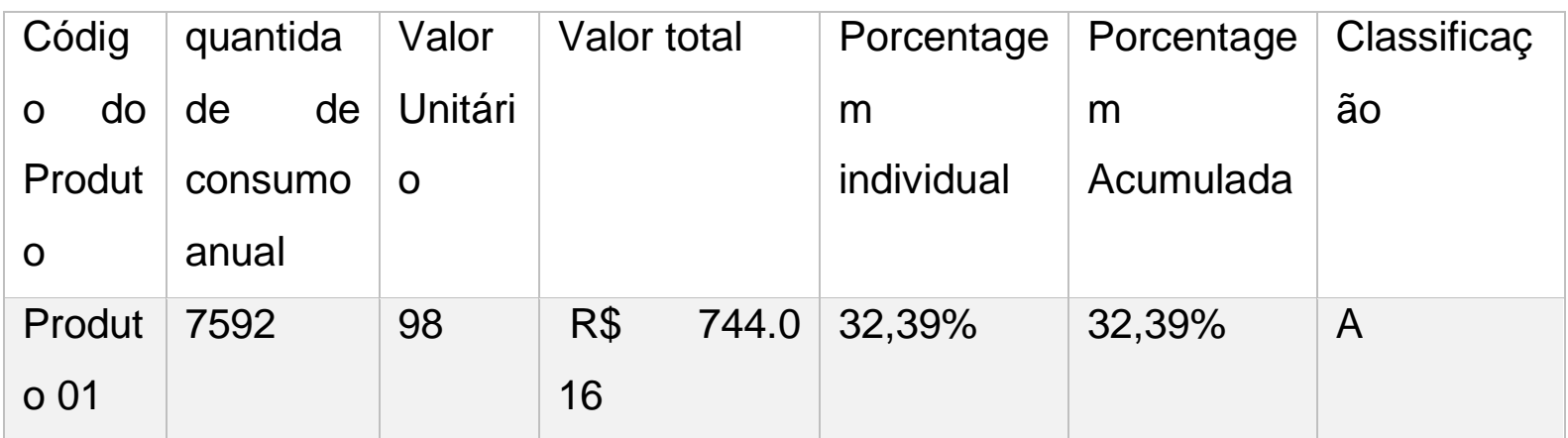




\begin{tabular}{|c|c|c|c|c|c|c|c|}
\hline $\begin{array}{l}\text { Produt } \\
002\end{array}$ & 1884 & 250 & $\begin{array}{l}\mathrm{R} \$ \\
00\end{array}$ & 471.0 & $20,51 \%$ & $52,90 \%$ & $A$ \\
\hline $\begin{array}{l}\text { Produt } \\
\text { o } 03\end{array}$ & 2331 & 194 & $\begin{array}{l}\mathrm{R} \$ \\
14\end{array}$ & 452.2 & $19,69 \%$ & $72,59 \%$ & $A$ \\
\hline $\begin{array}{l}\text { Produt } \\
\text { o } 04\end{array}$ & 700 & 350 & $\begin{array}{l}\mathrm{R} \$ \\
00\end{array}$ & 245.0 & $10,67 \%$ & $83,26 \%$ & $B$ \\
\hline $\begin{array}{l}\text { Produt } \\
\text { 0 } 05\end{array}$ & 729 & 200 & $\begin{array}{l}\mathrm{R} \$ \\
00\end{array}$ & 145.8 & $6,35 \%$ & $89,60 \%$ & $B$ \\
\hline $\begin{array}{l}\text { Produt } \\
\text { o } 06\end{array}$ & 747 & 100 & $\begin{array}{l}\mathrm{R} \$ \\
00\end{array}$ & 74.7 & $3,25 \%$ & $92,86 \%$ & $B$ \\
\hline $\begin{array}{l}\text { Produt } \\
\text { o } 07\end{array}$ & 2022 & 30 & $\begin{array}{l}\mathrm{R} \$ \\
60\end{array}$ & 60.6 & $2,64 \%$ & $95,50 \%$ & C \\
\hline $\begin{array}{l}\text { Produt } \\
\text { o } 08\end{array}$ & 136 & 286 & $\begin{array}{l}R \$ \\
96\end{array}$ & 38.8 & $1,69 \%$ & $97,19 \%$ & C \\
\hline $\begin{array}{l}\text { Produt } \\
\text { o } 09\end{array}$ & 330 & 100 & $\begin{array}{l}\mathrm{R} \$ \\
00\end{array}$ & 33.0 & $1,44 \%$ & $98,63 \%$ & C \\
\hline $\begin{array}{l}\text { Produt } \\
\text { o } 10\end{array}$ & 137 & 230 & $\begin{array}{l}R \$ \\
10\end{array}$ & 31.5 & $1,37 \%$ & $100,00 \%$ & C \\
\hline Total: & & & $\begin{array}{l}\mathbf{R} \$ \\
96\end{array}$ & 2.296 .7 & $100 \%$ & & \\
\hline
\end{tabular}

Fonte: Zenkova e Kabanova (2018), adaptada pelos autores. 
Gráfico 1 - Curva de classificação ABC de Zenkova e Kabanova (2018)

\section{Curva ABC}

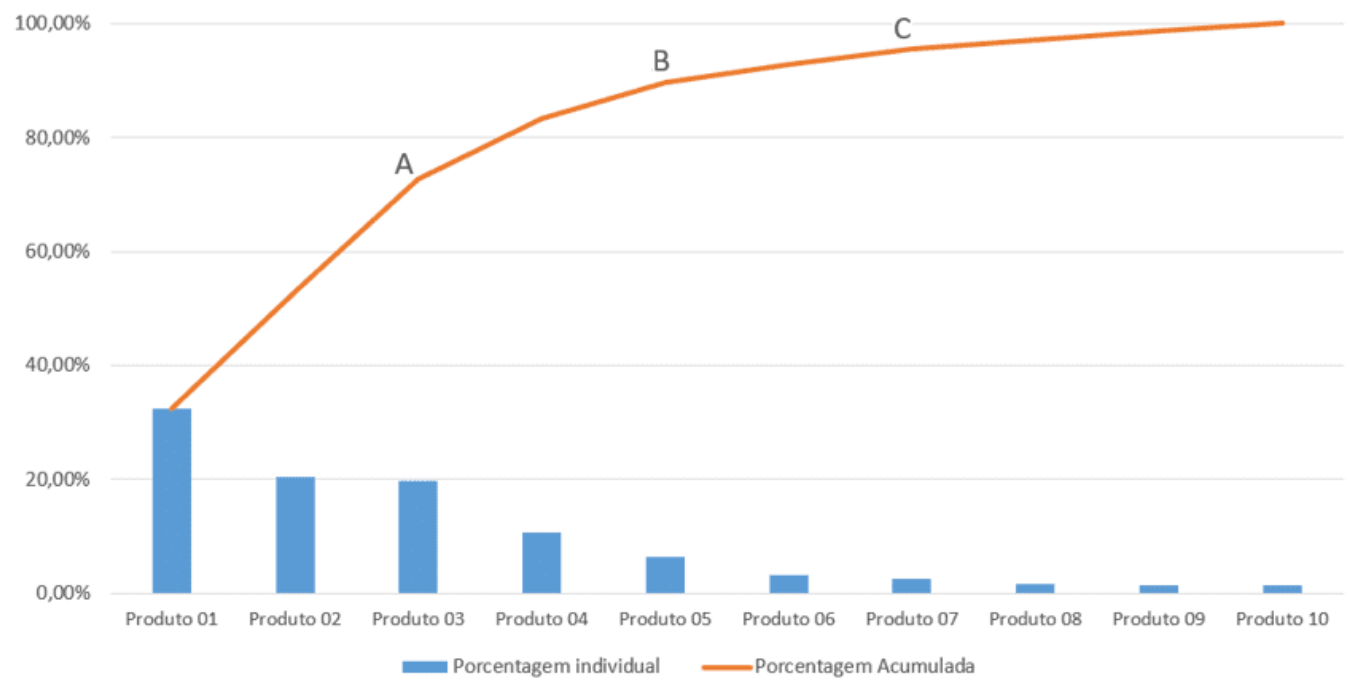

Fonte: elaborado pelos autores.

\section{METODOLOGIA DA PESQUISA}

O objetivo deste capítulo é discorrer acerca dos procedimentos metodológicos aplicados na presente pesquisa. O capítulo está dividido nos seguintes tópicos: delimitação da pesquisa; definição e método de pesquisa; universo e amostra; definição do instrumento de pesquisa.

\subsection{DELIMITAÇÃO DA PESQUISA}

Os materiais de consumo são elementos da despesa pública e se dividem em diversos subitens, no entanto a pesquisa se limita aos seguintes subitens:

- Material de expediente;

- Material de consumo de tecnologia da informação e comunicação; e

- Material de manutenção bens imóveis e instalações. 
A pesquisa utilizou dados da compra realizada no ano de 2018, uma vez que todo o processo de compras de 2019 ainda não estava concluído até o término do presente estudo.

\subsection{DEFINIÇÃO DE MÉTODO DE PESQUISA}

O presente trabalho adotou a forma de pesquisa quantitativa, e utilizou valores extraídos de empenhos e históricos do setor de armazenamento de materiais de consumo de um dos campi da instituição, bem como do portal compras governamentais.

\subsection{UNIVERSO E AMOSTRA}

A Instituição federal de ensino é composta por 8 campi, os quais serão o universo da pesquisa. Entretanto, os dados quantitativos da pesquisa serão retirados de apenas um dos campi, o qual servirá de pesquisa documental e amostra por acessibilidade, por estarem prontamente disponíveis para esse estudo.

O envolvimento dos demais campi, no atual trabalho, exigiria uma disposição maior de tempo e de coleta de informações, podendo, assim, ser uma sugestão para pesquisas futuras.

\subsection{DEFINIÇÃO DO INSTRUMENTO DE PESQUISA}

Foi realizado um levantamento das compras de materiais de consumo realizadas no ano de 2018, com base em dados quantitativos realizados por pesquisa documental. Por meio desses dados, foi realizada a classificação $A B C$ de materiais.

\section{RESULTADOS E DISCUSSÕES}

Este capítulo busca a apresentação, os comentários, bem como a interpretação dos dados quantitativos coletados pela pesquisa documental, a qual foi realizada em um dos oito campi da instituição federal de ensino superior. Ressalta-se que não foi 
possível a utilização dos dados dos demais campi, pois não haveria tempos para a coleta desses dados, devido ao afastamento social em decorrência da pandemia do COVID-19.

\subsection{ANÁLISE DE DADOS PARA A CLASSIFICAÇÃO ABC}

Gonçalves (2016) afirma que os percentuais na classificação $A B C$ não são rígidos. Segundo $o$ autor, eles podem ser alterados conforme o perfil da curva $A B C$. Nesse contexto, para a classificação $A B C$, após uma análise de conteúdo dos documentos do campus em estudo, foram adotados os seguintes parâmetros de classificação: os itens de classe A correspondem a $80 \%$ dos valores; os itens B a $15 \%$ dos valores; os itens $\mathrm{C}$ a $5 \%$ dos valores.

A classificação foi feita com 115 diferentes tipos de materiais de consumo adquiridos em 2018, para estocagem no almoxarifado, em um valor de $\mathrm{R} \$ 32.530,68$. Por meio da curva $A B C$, foi elaborada uma listagem com a ordem de prioridade de materiais adquiridos, nos quais o gestor de estoque deverá manter maior atenção. Observa-se que a após a classificação dos 115 materiais, foram obtidos 18 materiais para a classe A; 35 materiais para a classe $B$; e 62 materiais para a classe $C$, conforme mostra a gráfico 1 abaixo. 
Gráfico 2- Classificação $A B C$, quanto à quantidade de itens, para a compra realizada em 2018

\section{Quantidades de itens}

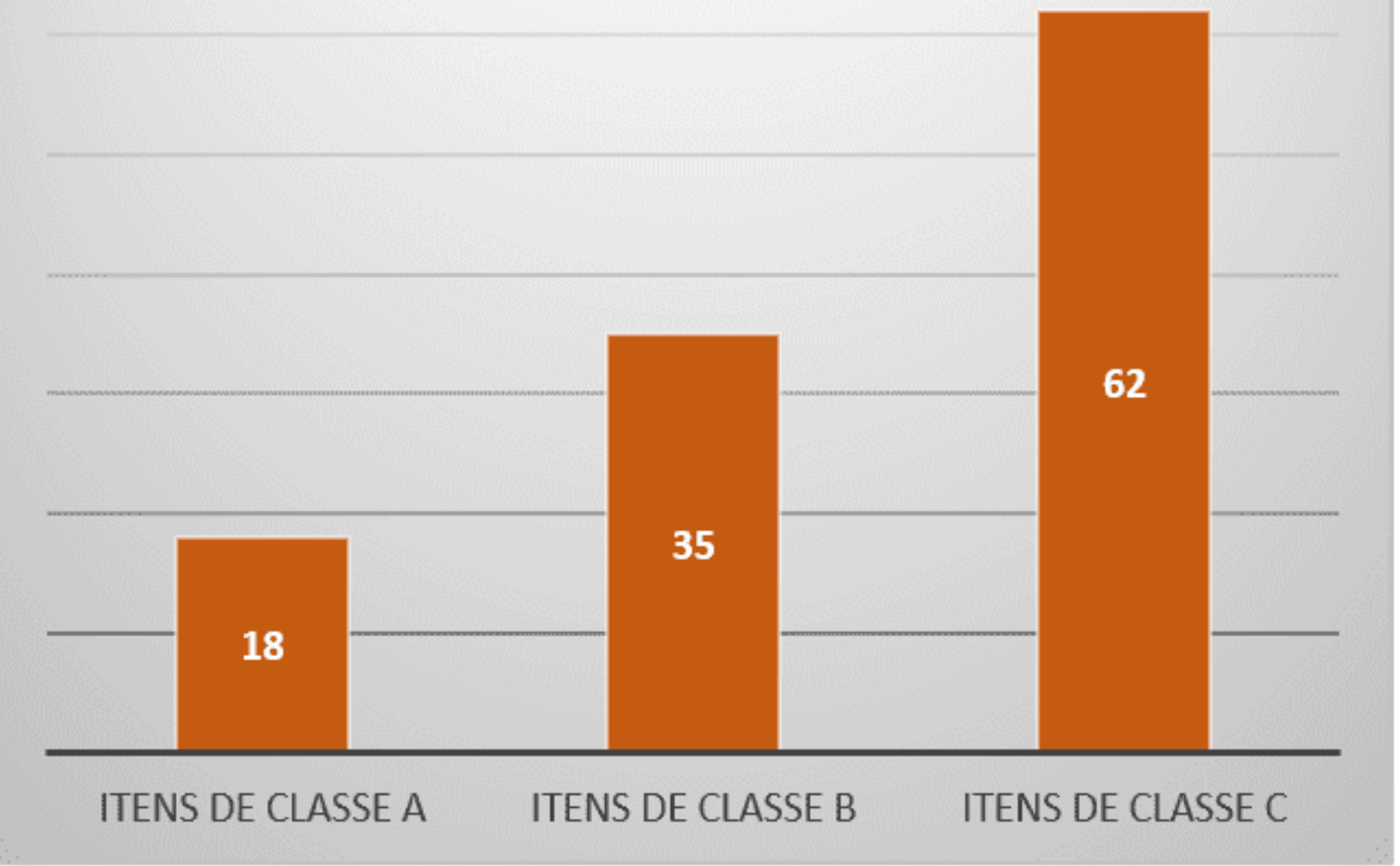

Fonte: elaborado pelo autor.

A classificação $A B C$ reafirmou que os itens de classe $A$ concentram os maiores recursos empregados na compra ( $R \$ 25.712,28)$; os recursos de médios valores $(R \$$ $5.188,56)$ foram classificados como $B$; e os materiais de classe $C$ concentraram os recursos de valores mais baixos ( $R \$ 1.629,84)$, conforme mostra o gráfico 2 abaixo. 
Gráfico 3 - Classificação ABC, quanto aos valores, para a compra realizada em 2018

\section{Valores dos íitens}

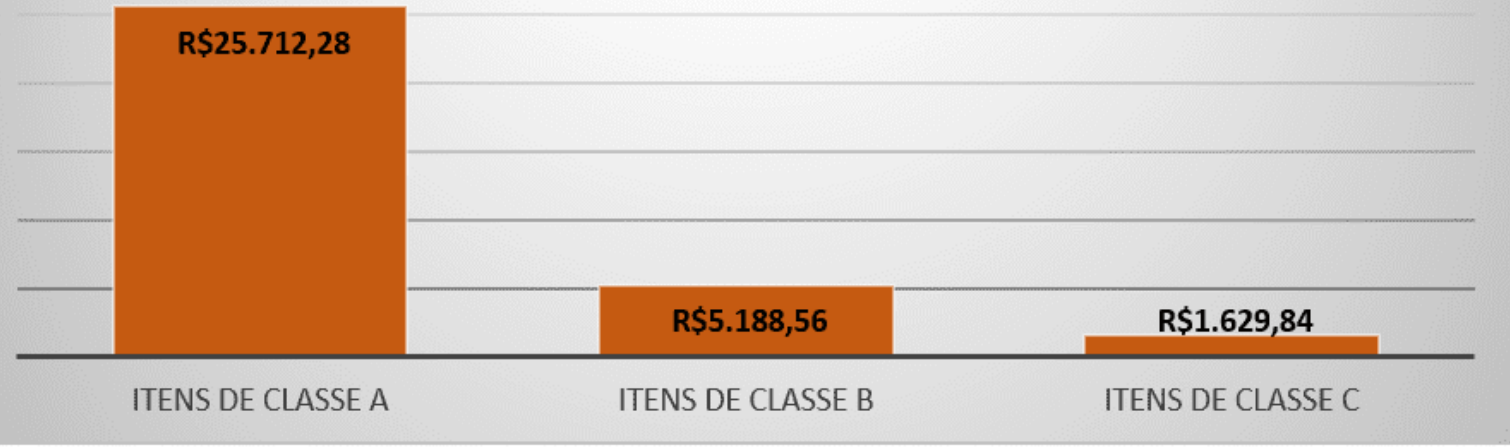

Fonte: elaborado pelos autores.

A tabela 1 abaixo traz alguns materiais como exemplo (de um total de 115 diferentes tipos de materiais adquiridos) em classificação $A B C$ da compra realizada pelo campus no ano de 2018.

Tabela 2 - Alguns exemplos da classificação ABC dos 115 diferentes tipos de materiais de consumo adquiridos

\begin{tabular}{|c|c|c|c|c|c|c|c|}
\hline $\begin{array}{l}\text { Códi } \\
\text { go do } \\
\text { Mater } \\
\text { ial }\end{array}$ & $\begin{array}{l}\text { Quantid } \\
\text { ade }\end{array}$ & $\begin{array}{l}\text { Valor } \\
\text { Unitário }\end{array}$ & Valor & total & $\begin{array}{l}\text { Porcenta } \\
\text { gem } \\
\text { individual }\end{array}$ & $\begin{array}{l}\text { Porcenta } \\
\text { gem } \\
\text { Acumula } \\
\text { da }\end{array}$ & $\begin{array}{l}\text { Classific } \\
\text { ação }\end{array}$ \\
\hline $\begin{array}{l}\text { Mater } \\
\text { ial } 01\end{array}$ & 800 & $\begin{array}{c}R \$ \\
16,39\end{array}$ & $\begin{array}{l}\mathrm{R} \$ \\
0\end{array}$ & $13.112,0$ & $40,31 \%$ & $40,31 \%$ & A \\
\hline$\ldots$ & $\ldots$ & $\ldots$ & $\ldots$ & & $\ldots$ & $\ldots$ & $\ldots$ \\
\hline $\begin{array}{l}\text { Mater } \\
\text { ial } 18\end{array}$ & 1 & $\begin{array}{cc}\mathrm{R} \$ & 3 \\
28,68\end{array}$ & $\begin{array}{l}\mathrm{R} \$ \\
8\end{array}$ & 328,6 & $1,01 \%$ & $79,04 \%$ & A \\
\hline $\begin{array}{l}\text { Mater } \\
\text { ial } 19\end{array}$ & 1 & $\begin{array}{c}\mathrm{R} \$ \\
26,91\end{array}$ & $\begin{array}{l}\mathrm{R} \$ \\
1\end{array}$ & 326,9 & $1,00 \%$ & $80,05 \%$ & B \\
\hline
\end{tabular}

RC: 62236

Disponível em: https://www.nucleodoconhecimento.com.br/engenharia-de-producao/classificacao$\underline{a b c}$ 


\begin{tabular}{|c|c|c|c|c|c|c|c|}
\hline $\begin{array}{l}\text { Mater } \\
\text { ial } 20\end{array}$ & 1 & $\begin{array}{c}\mathrm{R} \$ \\
18,00\end{array}$ & $\begin{array}{l}\mathrm{R} \$ \\
0\end{array}$ & 318,0 & $0,98 \%$ & $81,02 \%$ & B \\
\hline $\begin{array}{l}\text { Mater } \\
\text { ial } 21\end{array}$ & 2000 & $\begin{array}{c}\mathrm{R} \$ \\
0,16\end{array}$ & $\begin{array}{l}\mathrm{R} \$ \\
0\end{array}$ & 314,0 & $0,97 \%$ & $81,99 \%$ & $B$ \\
\hline$\cdots$ & $\cdots$ & $\cdots$ & $\ldots$ & & $\cdots$ & $\cdots$ & $\ldots$ \\
\hline $\begin{array}{l}\text { Mater } \\
\text { ial } 51\end{array}$ & 150 & $\begin{array}{c}\mathrm{R} \$ \\
0,46\end{array}$ & $\begin{array}{l}\mathrm{R} \$ \\
1\end{array}$ & 68,9 & $0,21 \%$ & $94,58 \%$ & B \\
\hline $\begin{array}{l}\text { Mater } \\
\text { ial } 52\end{array}$ & 15 & $\begin{array}{c}\mathrm{R} \$ \\
4,51\end{array}$ & $\begin{array}{l}\mathrm{R} \$ \\
5\end{array}$ & 67,6 & $0,21 \%$ & $94,78 \%$ & $B$ \\
\hline $\begin{array}{l}\text { Mater } \\
\text { ial } 53\end{array}$ & 24 & $\begin{array}{c}R \$ \\
2,79\end{array}$ & $\begin{array}{l}\mathrm{R} \$ \\
6\end{array}$ & 66,9 & $0,21 \%$ & $94,99 \%$ & B \\
\hline $\begin{array}{l}\text { Mater } \\
\text { ial } 54\end{array}$ & 12 & $\begin{array}{c}\mathrm{R} \$ \\
5,16\end{array}$ & $\begin{array}{l}R \$ \\
2\end{array}$ & 61,9 & $0,19 \%$ & $95,18 \%$ & C \\
\hline $\begin{array}{l}\text { Mater } \\
\text { ial } 55\end{array}$ & 30 & $\begin{array}{c}R \$ \\
1,86\end{array}$ & $\begin{array}{l}\mathrm{R} \$ \\
0\end{array}$ & 55,8 & $0,17 \%$ & $95,35 \%$ & C \\
\hline $\begin{array}{l}\text { Mater } \\
\text { ial } 56\end{array}$ & 50 & $\begin{array}{c}\mathrm{R} \$ \\
1,10\end{array}$ & $\begin{array}{l}\mathrm{R} \$ \\
0\end{array}$ & 55,0 & $0,17 \%$ & $95,52 \%$ & C \\
\hline $\begin{array}{l}\text { Mater } \\
\text { ial } 57\end{array}$ & 50 & $\begin{array}{c}\mathrm{R} \$ \\
1,10\end{array}$ & $\begin{array}{l}\mathrm{R} \$ \\
0\end{array}$ & 55,0 & $0,17 \%$ & $95,69 \%$ & C \\
\hline $\begin{array}{l}\text { Mater } \\
\text { ial } 58\end{array}$ & 2 & $\begin{array}{c}\mathrm{R} \$ \\
27,08\end{array}$ & $\begin{array}{l}R \$ \\
6\end{array}$ & 54,1 & $0,17 \%$ & $95,86 \%$ & C \\
\hline$\cdots$ & $\cdots$ & $\cdots$ & $\ldots$ & & $\ldots$ & $\cdots$ & ... \\
\hline $\begin{array}{l}\text { Mater } \\
\text { ial } \\
111\end{array}$ & 5 & $\begin{array}{c}\mathrm{R} \$ \\
1,02\end{array}$ & $\begin{array}{l}\mathrm{R} \$ \\
0\end{array}$ & 5,1 & $0,02 \%$ & $99,95 \%$ & C \\
\hline $\begin{array}{l}\text { Mater } \\
\text { ial } \\
112\end{array}$ & 5 & $\begin{array}{c}\mathrm{R} \$ \\
0,95\end{array}$ & $\begin{array}{l}\mathrm{R} \$ \\
5\end{array}$ & 4,7 & $0,01 \%$ & $99,97 \%$ & C \\
\hline
\end{tabular}




\begin{tabular}{|l|l|l|l|l|l|l|l|l|}
\hline $\begin{array}{l}\text { Mater } \\
\text { ial }\end{array}$ & 3 & $\mathrm{R} \$$ & $\mathrm{R} \$$ & 4,0 & $0,01 \%$ & $99,98 \%$ & $\mathrm{C}$ \\
\hline 113 & 1,36 & 8 & & & & \\
\hline $\begin{array}{l}\text { Mater } \\
\text { ial } \\
114\end{array}$ & 3 & $\mathrm{R} \$$ & $\mathrm{R} \$$ & 3,6 & $0,01 \%$ & $99,99 \%$ & $\mathrm{C}$ \\
\hline $\begin{array}{l}\text { Mater } \\
\text { ial }\end{array}$ & 2 & $\mathrm{R} \$$ & 6 & & & & \\
\hline $\begin{array}{l}115 \\
\text { TOTAL: }\end{array}$ & 1,80 & 0 & & & & & & \\
\hline
\end{tabular}

Fonte: elaborada pelos autores.

As compras de materiais de consumo que são direcionados ao almoxarifado do campus utilizado como pesquisa documental são muito diversificadas e extensas, o que ocasiona muitos materiais comprados e não foram utilizados nesta pesquisa.

O elemento da despesa denominado material de consumo possui diversos subitens, entretanto, a pesquisa se limitou ao estudo dos materiais de consumo, cujos subitens sejam:

- Material de expediente;

- Material de consumo de tecnologia da informação e comunicação; e

- Material de manutenção bens imóveis e instalações.

Dentre os 115 materiais estudados, foi possível observar a existência de 18 materiais de consumo em que a gestão do almoxarifado deverá focar na compra para o próximo período. Uma vez que são materiais com pouca quantidade adquiridas e máximo valor despendido. 
Tabela 3 - Classificação ABC dos 115 materiais de consumo estudados

\begin{tabular}{|l|l|lr|}
\hline Classificação & Materiais diferentes & Valores \\
\hline $\mathrm{A}$ & 18 & $\mathrm{R} \$$ & $25.712,28$ \\
\hline $\mathrm{B}$ & 35 & $\mathrm{R} \$$ & $5.188,56$ \\
\hline $\mathrm{C}$ & 62 & $\mathrm{R} \$$ & $1.629,84$ \\
\hline TOTAL & $\mathbf{1 1 5}$ & $\mathbf{R} \$$ & $\mathbf{3 2 . 5 3 0 , 6 8}$ \\
\hline
\end{tabular}

Fonte: elaborada pelos autores.

\section{CONCLUSÕES E CONSIDERAÇÕES FINAIS}

Este trabalho buscou classificar os materiais de consumo para determinar os materiais em que a gestão deverá manter a máxima atenção durante o planejamento da demanda de compras em uma instituição federal de ensino superior. Desse modo, o objetivo foi alcançado por meio da classificação $A B C$ ou curva de Pareto.

Dentre os 115 materiais de consumo estudado, 18 materiais foram identificados como os de máxima importância para a gestão do almoxarifado, uma vez que eles concentram os maiores valores destinados para esse elemento da despesa pública. Desse modo, controles como estimativas de compras, prioridades na aquisição e valores empreendidos poderão ser realizados com mais atenção nesses 18 diferentes tipos de materiais de consumo classificados como A.

\subsection{LIMITAÇÕES DA PESQUISA}

Algumas limitações foram encontradas, embora não tenham impedido a continuidade da pesquisa. Nesse sentido, destacam-se o controle de estoque dos demais campi não poderem ser utilizado no estudo, o grande número de subitens de materiais de consumo a ser estudado, o ciclo de compra de 2019 muito demorado e ainda em andamento até a conclusão desta pesquisa, bem como pandemia do COVID-19 que impossibilitou o acesso in loco aos documentos do almoxarifado. 


\subsection{PROPOSTA PARA TRABALHOS FUTUROS}

Como proposta para trabalhos futuros o estudo pode ser realizado nos demais campi da instituição, bem como com os demais subitens de materiais de consumo.

\subsection{PRINCIPAIS ACHADOS}

Os processos de compras na instituição de ensino são demasiadamente longos, com início em determinado ano e não são totalmente concluídos até o término do ano subsequente.

\section{REFERÊNCIAS}

AMRAN, T. G.; FATIMA, Z. Lagrange multiplier for perishable inventory model considering warehouse capacity planning. AIP Conference Proceedings, v. 1855, n. January, 2017.

CEDILLO-CAMPOS, M. G.; CEDILLO-CAMPOS, H. O. W@reRISK method: Security risk level classification of stock keeping units in a warehouse. Safety Science, v. 79, p. 358-368, 2015.

DIAS, Marco Aurélio P. Administração de Materiais/Princípios, Conceitos e Gestão. 6a. Edição. São Paulo: Editora Atlas, 2010.

EDUARDO, A.; UFSM, M. Aplicação do método de criticidade de materiais em estoques hospitalares. Encontro Nacional de Engenharia de Produção, n. 1990, p. 849-856, 2004.

FENG, Y. et al. A Distributed Procurement Cost Control Scheme of Medical Materials for Regional Medical Consortiums. 2018.

GONÇALVES, Paulo Sérgio. Administração de Materiais. 5를 Edição. Rio de Janeiro: Editora Campus, 2016. 
HALASZ, M. T. Análise de demandas de camadas de proteção definidas com a metodologia lopa utilizando um sistema de gestão de ativos em instrumentação. Dissertação (Mestrado em Sistemas de Gestão) - Programa de Pós-Graduação em Sistemas de Gestão, Universidade Federal Fluminense, 2017.

ISHIZAKA, A. et al. DEASort: Assigning items with data envelopment analysis in ABC classes. International Journal of Production Economics, v. 199, n. January, p. 7$15,2018$.

JEMELKA, M.; CHRAMCOV, B.; KŘIŽ̌, P. ABC analyses with recursive method for warehouse. 2017 4th International Conference on Control, Decision and Information Technologies, CoDIT 2017, v. 2017- Janua, p. 960-963, 2017.

LIU, Z. The anti-hepatitis drug use effect and inventory management optimization from the perspective of hospital drug supply chain. Pakistan journal of pharmaceutical sciences, v. 30, p. 1917-1922, 1 set. 2017.

LOSILLA, Fabrício Aparecido Cano; VALENTE, Carlos Magno de Oliveira. Análise de Sistema de Gestão de Estoques por Meio de Curva ABC e Inventário Cíclico: Estudo de Caso em uma Industria no Setor Automobilístico. Revista Científica Multidisciplinar Núcleo do Conhecimento. Edição 08. Ano 02, Vol. 03. pp 05-17, 2017.

PRAJAKSUWITHEE, S.; CHUTIMA, P. Inventory Management System Development in a Flexible Printed Circuit Board Manufacturer. 2019 IEEE 6th International Conference on Industrial Engineering and Applications, ICIEA 2019, p. 246-250, 2019.

SANTOS et al. Cálculo da previsão de demanda de uma multinacional Calculation. Brazilian Journal of Development, v. 4, n. 6, p. 3035-3052, 2018. 
ZENKOVA, Z.; KABANOVA, T. The ABC-XYZ analysis modified for data with outliers. Proceedings - GOL 2018: 4th IEEE International Conference on Logistics Operations Management, p. 1-6, 2018.

VIANNA, João José. Administração de Materiais/Um enfoque Prático. 1ª Edição. São Paulo: Editora Atlas, 2006.

ZHAO, J.; DONG, W. the Total Cost Inventory System Optimization. v. 65, p. 344347, 2016.

Enviado: Setembro, 2020.

Aprovado: Outubro, 2020. 